\title{
Das Programm P-5 „Wissenschaftliche Information: Zugang, Verarbeitung und Speicherung“: teilnehmeroffene Dienste der Hochschulen, für die Hochschulen
}

\begin{abstract}
Von 2013 bis 2017 fliessen über das Programm „Wissenschaftliche Information“ von swissuniversities CHF 75 Mio. projektgebundene Beiträge des Bundes in den Aufbau eines Servicenetzwerks für digitale wissenschaftliche Information. Das Servicenetzwerk soll von den Schweizer Hochschulen über eine Koordinationsstelle gemeinsam betrieben und weiterentwickelt werden. Der Beitrag beschreibt den Hintergrund, die Absicht und den aktuellen Stand des Programms. Dabei wird besonders auf diejenigen Bereiche des Programms eingegangen, in denen die Hochschulbibliotheken eine Schlüsselrolle spielen.
\end{abstract}

\section{Projektgebundene Beiträge zur Förderung des wissenschaftlichen Arbeitens mit digitaler Information}

Das Programm „Wissenschaftliche Information: Zugang, Verarbeitung und Speicherung“ baute 2013 auf zwei Vorgängerprogrammen auf: e-lib.ch, das unter dem Lead der ETH-Bibliothek erfolgreich digitale Infrastrukturen im bibliotheksnahen Bereich lancierte, und AAA/SWITCH. ${ }^{1}$ Mit AAA/SWITCH baute der Netzwerkanbieter der Schweizer Hochschulen, SWITCH, IT-Infrastrukturen in den Bereichen E-Learning, Grid Computing und virtuelle Organisationen auf. Das neue Programm erhielt den Auftrag, kooperative Dienste im Bereich der wissenschaftlichen Information als übergreifendes Aufgabenfeld der Bibliotheken und der ITDienstleister (IT-Services, Scientific IT, SWITCH) der Hochschulen zusammenzuführen. 2017 steht „Wissenschaftliche Information“ am Beginn einer zweiten Finanzierungsperiode. Bis 2020 sollen die aufgebauten Dienste zu einem Servicenetzwerk konsolidiert werden. Für dieses Netzwerk soll eine bleibende Koordina-

1 Vgl. http://www.e-lib.ch und https://projects.switch.ch/de/aaa/. Alle Links in diesem Beitrag wurden am 26.03.2018 überprüft.

Ә Open Access. (C) Gabi Schneider, publiziert von De Gruyter. (c) BY-NC-ND Dieses Werk ist lizenziert unter der Creative Commons Attribution-NonCommercial-NoDerivatives 4.0 Lizenz. 
tionsstelle geschaffen werden, die als permanente Drehscheibe für den Vertrieb und den Anschub gemeinsamer Dienste der Hochschulen im Bereich der digitalen wissenschaftlichen Information dient. ${ }^{2}$

Von 2013 bis 2016 lief das Programm unter der administrativen Nummer SUK P-2, von 2017 bis 2020 ist es P-5, das Programm Nr. 5 in dieser Periode. Der Wegfall des Kürzels „SUK“ (für die ehemalige Schweizerische Universitätskonferenz) ist Ausdruck einer Änderung der gesetzlichen Grundlagen. 2015 trat das Hochschulförderungs- und -koordinationsgesetz (HFKG) in Kraft, das die Zusammenarbeit zwischen Bund und Kantonen im Hochschulbereich neu regelt. ${ }^{3}$ In diesem Kontext wurden 2015 die bis dahin separaten nationalen Gremien der universitären Hochschulen und der ETH, der Fachhochschulen und der Pädagogischen Hochschulen zusammengelegt. Die drei Rektorenkonferenzen sind seither im Verein swissuniversities unter einem gemeinsamen Dach zusammengefasst. Als Legislative fungiert neu die Schweizerische Hochschulkonferenz (SHK). Der Schweizerische Akkreditierungsrat und seine Agentur sorgen für die Qualitätssicherung im Hochschulbereich.

Seit Anfang 2017 sind auch die Finanzierungsbestimmungen des HFKG in Kraft. Neben den Grundbeiträgen und den Bauinvestitions- und Baunutzungsbeiträgen kommt den Hochschulen Finanzhilfe des Bundes weiterhin in Form projektgebundener Beiträge zu. Gemäss Art. 59 HFKG unterstützt der Bund mit diesem Instrument Aufgaben von gesamtschweizerischer, hochschulpolitischer Bedeutung. Die Inhalte werden von den Hochschulen vorgeschlagen und von swissuniversities koordiniert bei der SHK eingereicht. Der Hochschulrat der SHK, in dem Bund und Kantone gemeinsam vertreten sind, entscheidet über die Umsetzung. Mit CHF 45 Mio. in der Beitragsperiode 2013 bis 2016 und CHF 30 Mio. in der Periode 2017 bis 2020 gehört „Wissenschaftliche Information“ zu den höchstdotierten Umsetzungsbereichen. ${ }^{4}$ Die Verantwortung für die Durchführung des Programms liegt bei swissuniversities.

2 Alle Grundlagendokumente des Programms sind auf der Webseite verfügbar: http://www.swis suniversities.ch/isci. Der Beitrag orientiert sich an: Gabi Schneider: Wissenschaftliche Information: Zugang, Verarbeitung und Speicherung. Ein Förderprogramm zur Bündelung der wissenschaftlichen Information in der Schweiz. In: BIBLIOTHEK - Forschung und Praxis 40/3 (2016), S. 424-433.

3 SR 414.20, Bundesgesetz über die Förderung der Hochschulen und die Koordination im schweizerischen Hochschulbereich (Hochschulförderungs- und -koordinationsgesetz, HFKG) (https:// www.admin.ch/opc/de/classified-compilation/20070429/index.html).

4 Vgl. die Informationen auf den Webseiten der Schweizerischen Hochschulkonferenz: https:// www.shk.ch/themen/projektgebundene-beitraege/. 
Projektgebundene Beiträge werden befristet ausgerichtet. Förderung erfolgt nach dem Prinzip der Anschubfinanzierung: Projekte, die dem vorgegebenen Rahmen entsprechen und im Evaluationsverfahren bestehen, erhalten 50\% der Projektkosten (matching funds). Aus geförderten Vorhaben sollen eigenständige Infrastrukturen entstehen.

\section{Strategische Grundlagen}

Die nationale Strategie des Programms, die von der damaligen SUK beauftragt und im April 2014 bewilligt wurde, postuliert die „Bündelung der Kräfte in der wissenschaftlichen Information“. ${ }^{5}$ Danach legt der grosse Veränderungsdruck im Bereich der digitalen wissenschaftlichen Information ein gemeinsames Handeln der Schweizer Hochschulen nahe. Der Aufbau eines Netzwerks von Diensten, die allen Hochschulen offenstehen, soll zu Synergien und Effizienzgewinnen führen. Das starke Kostenwachstum soll begrenzt und die Wettbewerbsfähigkeit der Schweiz als Spitzenstandort für Bildung und Forschung gestärkt werden. Eine anerkannte Organisation soll das Servicenetzwerk koordinieren und den dauerhaften Betrieb von Diensten gewährleisten.

Die Dienste des Servicenetzwerks sollen den einfachen Zugang zu Publikationen und Daten ermöglichen und Werkzeuge für deren Bearbeitung und Speicherung anbieten. Plangemäss soll Forschenden, Lehrenden und Lernenden in der Schweiz bis 2020 ein umfangreiches Grundangebot an digitalen Inhalten von wissenschaftlicher Relevanz zur Verfügung stehen. Verfügbarkeit auf nationaler Ebene, Stabilität, Agilität und Wettbewerbsfähigkeit sollen die Dienste auszeichnen.

\section{SUK P-2 (2013-16): Aufbau von Diensten in vier Hauptstossrichtungen}

In der Phase SUK P-2 konnte das Programm CHF 37 Mio. Fördermittel für Projekteingaben zur Verfügung stellen. ${ }^{6}$ In einem breit angelegten Bottom up-Prozess wurden 2013 die Umsetzungsstrategie und das Projektauswahlverfahren erarbei-

5 SUK P-2: Bündelung der Kräfte in der wissenschaftlichen Information: nationale Strategie. Von der SUK bewilligt am 03.04.2014, (vgl. Grundlagendokumente, wie Anm. 2).

6 SUK P-2 (2013-2016) verfügte insgesamt über Mittel in Höhe von CHF 45,3 Mio. Davon wurden CHF 8 Mio. für die Weiterführung von Vorgängerinitiativen bis zur Bewilligung der Strategie und 
tet. Das White Paper for a Swiss Information Provisioning and Processing Infrastructure 2020 beschreibt die strategische Ausgangslage und die Prozesse. Es schlug den Beitragsberechtigten schliesslich 39 konkrete Umsetzungsmassnahmen für Projekteingaben vor. Diese wurden vier strategischen Hauptstossrichtungen zugeordnet, die in P-5 weiter bestehen: „Publikationen“, „eScience“, „Basis“ und „Dienste“. Abbildung 1 illustriert, welche Umsetzungsbereiche des Programms in welcher Hauptstossrichtung zusammengefasst sind. ${ }^{7}$

\section{Vier Hauptstossrichtungen}

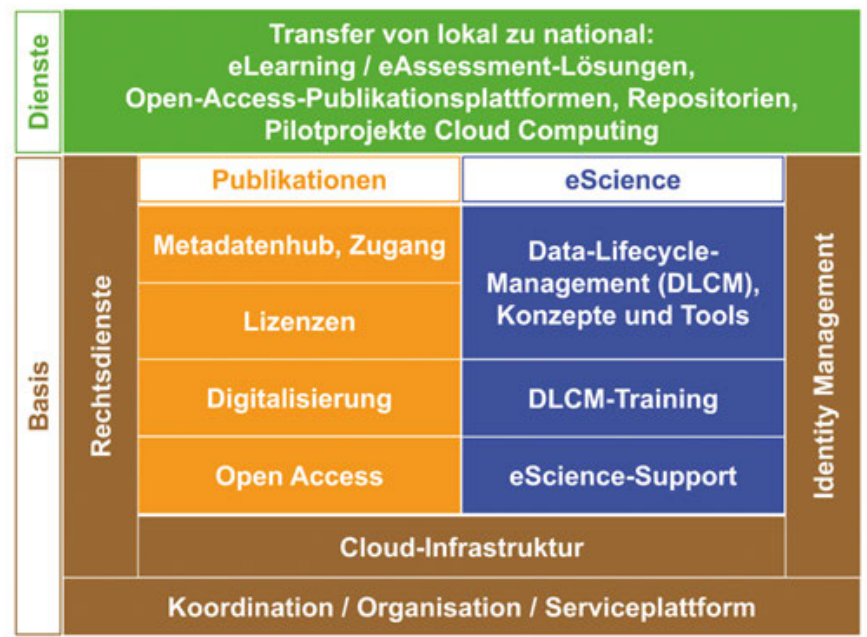

Abb. 1: Hauptstossrichtungen und Inhalte des Programms (@ Programmleitung P-5)

- In der Hauptstossrichtung „Publikationen“ soll durch die Erweiterung von Lizenzen und die Förderung von Digitalisierung und Open Access das elektronische Grundangebot an wissenschaftlichen Publikationen vergrössert

für die Programmorganisation eingesetzt. Mittel aus SUK P-2 können bis Ende 2018 verwendet werden.

7 Für die komplette Liste der Umsetzungsmassnahmen vgl. SUK P-2: White Paper for a Swiss Information Provisioning and Processing Infrastructure 2020. 2014, hier Kapitel 5.3. Eine zusammenfassende Beschreibung der Hauptstossrichtungen liefert die nationale Strategie, „Bündelung der Kräfte in der wissenschaftlichen Information“, Kapitel 2.4 bis 2.8 (vgl. Grundlagendokumente, wie Anm. 2). 
werden. Ergänzend werden Suchlösungen und der Aufbau von Metadatenhubs gefördert.

- Bei „eScience“ steht die Bewirtschaftung von Forschungsdaten im Zentrum. Hier sollen institutionsübergreifend standardisierte Prozesse für den Zugang, die Bearbeitung, die Nachnutzung und die Archivierung von Forschungsdaten entwickelt werden. Trainings und Supportdienste sollen Forschende dabei unterstützen, Methoden und Knowhow den verfügbaren Rechenleistungen entsprechend zu skalieren.

- „Basis“ fördert den Aufbau technischer und organisatorischer Grundlagen wie Cloud-Infrastrukturen, ein institutionsunabhängiges Identity-Management, Rechtsdienste, aber auch die Serviceplattform für den künftigen Vertrieb der aufgebauten Dienste.

- Die Hauptstossrichtung „Dienste“ soll Anreize setzen, bestehende Dienste wie Repositorien, Publikationsplattformen oder E-Learning-Werkzeuge für weitere Hochschulen zu öffnen und zu nationalen Diensten zu entwickeln.

Der Lenkungsausschuss erhielt zudem die Möglichkeit, Umsetzungsmassnahmen durch die Vergabe von Mandaten gezielt anzustossen.

Die Erarbeitung der strategischen Grundlagen, ihre konsequente Umsetzung bei der Projektauswahl und die Verankerung des Programms nahmen rund zwei Jahre in Anspruch. Erst seit Sommer 2015 ist die Grundlage für eine schrittweise Konsolidierung der geförderten Dienste gegeben. Abbildung 2 bilanziert den Erfolg der Projekteingaben und die Vergabe von Fördermitteln in SUK P-2. Waren in der ersten Eingabe im April 2014 nur fünf von 42 Anträgen bewilligt worden, pendelte sich die Erfolgsquote nach vier Evaluationen bei ca. 30\% ein. Insgesamt wurden 29\% der Projektanträge nach der Erst- oder Zweitbeurteilung bewilligt. Bis Ende 2016 förderte das Programm 23 Projekte. Die neun Anträge, die im Herbst 2016 eingereicht wurden, betrafen mit zwei Ausnahmen Projekte zur Fertigstellung oder Weiterentwicklung bereits geförderter Dienste.

Um den Aufbau von Diensten nicht zu bremsen, konnten in Absprache mit dem Staatssekretariat für Bildung, Forschung und Innovation (SBFI) ab 2015 Projekte mit Laufzeiten über 2016 hinaus bewilligt werden, unter Vorbehalt der Weiterführung des Programms in der Periode 2017 bis 2020. Die Bilanz Ende 2016 zeigt, dass rund CHF 8 Mio. der bewilligten Fördermittel zu Lasten von P-5 gehen. 


\section{Bilanz nach der sechsten Eingabefrist}

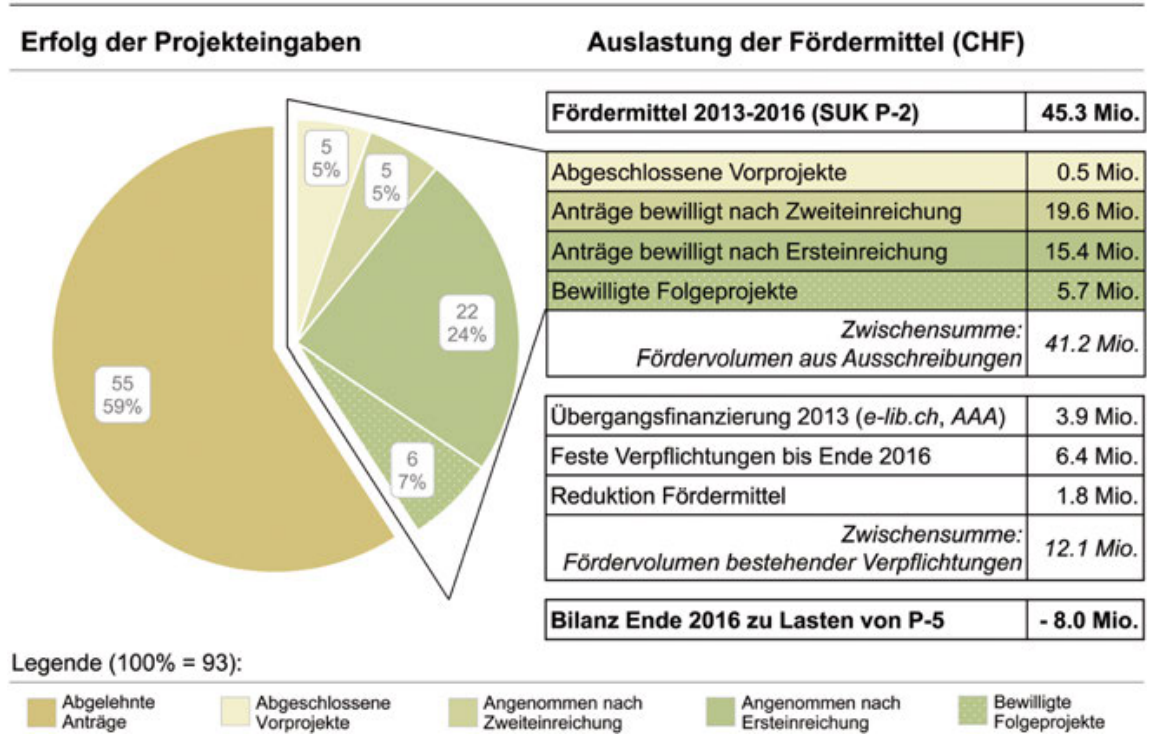

Abb. 2: Auswertung der Projekteingaben bis Ende 2016 (@ Programmleitung P-5)

\section{P-5 (2017-2020): Konsolidierung und Serviceorientierung}

Im Mai 2016 bewilligte der Hochschulrat der SHK die Fortsetzung des Programms mit gleichbleibendem Titel und neuer Nummer. ${ }^{8}$ In einer Ex-ante-Evaluation der Vorhaben für projektgebundene Beiträge 2017 bis 2020 hatte die Fortsetzung des Programms Anfang 2015 die höchste Punktzahl bezüglich Systemrelevanz erhalten. Diese wurde mit Vorteilen im internationalen Wettbewerb, der Generierung zusätzlicher Steuerungsinformationen und einem grösseren gesellschaftlichen Bedürfnis deutlich bejaht. Das Programm wurde als Aufgabe von gesamtschweizerischer hochschulpolitischer Bedeutung gemäss Artikel 59 HFKG gewertet. „Wissenschaftliche Information“ soll die Bildung von Kompetenzzentren fördern und mittelfristig eine Portfoliobereinigung ermöglichen. ${ }^{9}$

8 Projektantrag „Wissenschaftliche Information: Zugang, Verarbeitung und Speicherung“ vom 16.02.2016.

9 Andrea Schenker-Wicki: Projektgebundene Beiträge nach HFKG. Ex-ante-Evaluation der von den Schweizerischen Hochschulen eingegebenen Projekte für die Beitragsperiode 2017-2020. 2015, S. 13-14 (internes Dokument). 
P-5 wurde mit einem Budget von CHF 30 Mio. ausgestattet. Im Anschluss an die Bewilligung aktualisierte das Programm die für SUK P-2 definierten Umsetzungsmassnahmen und veröffentlichte Ende November die Umsetzungsstrategie 2017 bis 2020, die das White Paper als Grundlage für Projektanträge ablöste. ${ }^{10}$ Im Mittelpunkt stehen nun die Konsolidierung der aufgebauten Dienste, ihre Formierung zu einem Servicenetzwerk und der Übergang der Programmorganisation in eine Koordinationsstelle für das Netzwerk. Der Schwerpunkt der Programmorganisation verlagert sich von der Akquisition in Richtung Evaluation, Servicemanagement und Organisationsentwicklung. In den kommenden Jahren soll die Wirkung des Programms erhöht und sein Nutzen quantitativ nachweisbar gemacht werden. Unter dem Titel „Umsetzungsmassnahmen für den Ausbau und die Stärkung von Services“ wurde den vier Hauptstossrichtungen eine Reihe von Massnahmen vorangestellt, die den Ausbau von Servicekomponenten unterstützen. An erster Stelle steht die Förderung der Öffnung eines Dienstes durch die Übernahme der Investitionskosten bzw. der Zutrittskosten eines neuen Teilnehmers. Einen Antrag stellen können alle Dienstleister, die fachlich ins Spektrum des Programms fallen.

Nach Abzug der erwähnten Verpflichtungen aus SUK P-2 in Höhe von CHF 8 Mio. und dem jährlichen Aufwand für die Programmorganisation in Höhe von insgesamt CHF 4 Mio. stehen P-5 - unter Vorbehalt von Kürzungen CHF 18 Mio. für die Projektförderung zur Verfügung. Bei der Mittelvergabe wird der Lenkungsausschuss vermehrt qualitative und strategische Überlegungen gegeneinander abwägen müssen. Er wird auch darüber entscheiden müssen, in welchem Umfang bereits geförderte Dienste weitere Mittel erhalten und welcher Betrag für neue Vorhaben frei bleibt.

\section{Zum Entwicklungsstand, unter besonderer Berücksichtigung der Bibliotheken}

Im Strategieprozess für SUK P-2 wurde die in Abbildung 3 dargestellte Matrix aufgebaut. Sie macht sichtbar, in welchen Hauptstossrichtungen (Y-Achse) Umsetzungsmassnahmen für die im Programmantrag für SUK P-2 ${ }^{11}$ beschriebenen Handlungsfelder (X-Achse) angeboten wurden. Die Matrix diente in der Folge der

10 P-5: Umsetzungsstrategie 2017 bis 2020. 2016 (vgl. Grundlagendokumente, wie Anm. 2).

11 Projektgebundene Beiträge 2013-2016: Antrag auf ein SUK-Programm. 2012. Das Dokument ist im Archiv der Programmwebseite verfügbar (vgl. Grundlagendokumente, wie Anm. 2). 
Illustration des Programmfortschritts. Abbildung 3 zeigt den Umsetzungsstand des Programms Ende 2016. Die bewilligten Projekte sind im entsprechenden Feld der Matrix eingetragen mit Angabe der Umsetzungsmassnahme aus dem White Paper, die sie adressieren. ${ }^{12}$

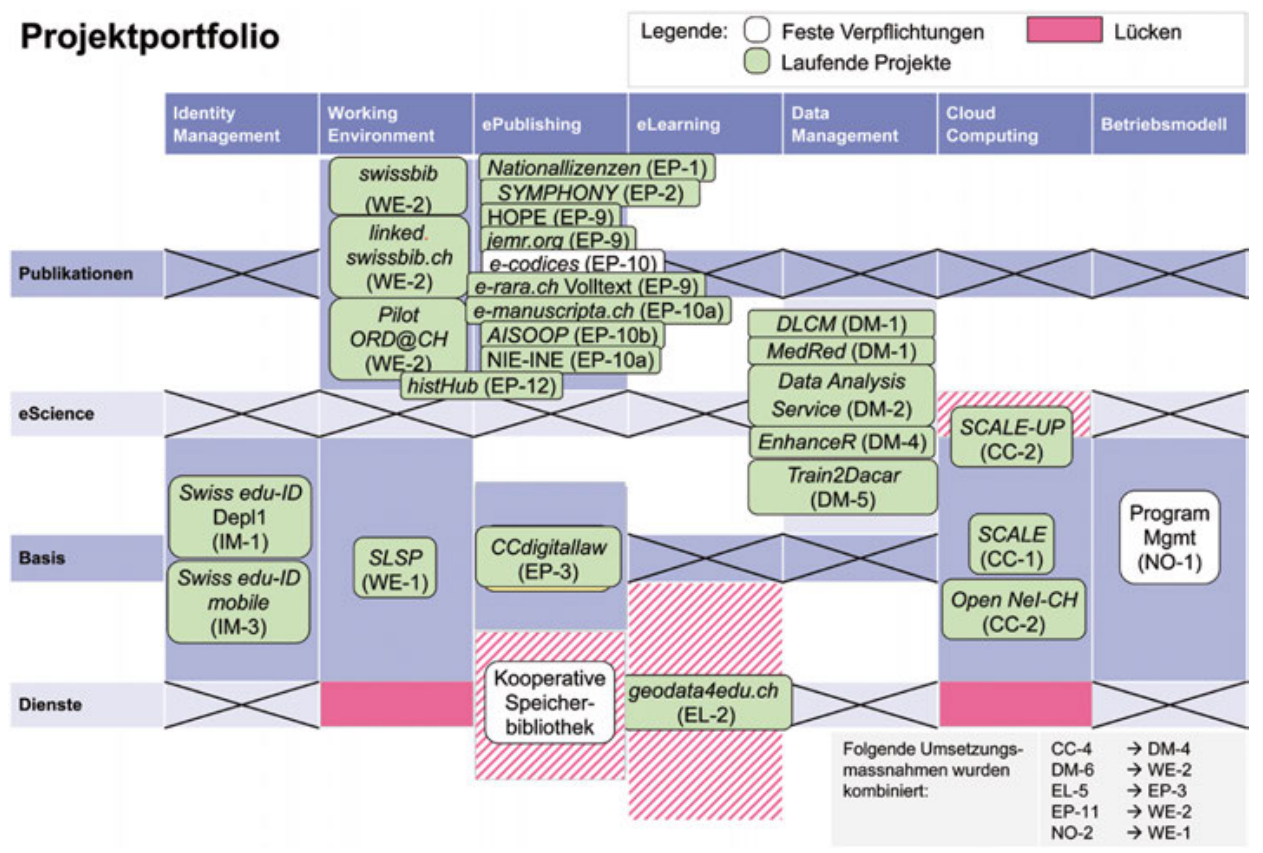

Abb. 3: Projektmatrix, Stand 31. Dezember 2016 (৫ Programmleitung P-5)

Die strategischen Bereiche, in denen Ende 2016 keine bzw. nur wenige Dienste gefördert wurden, sind durch die roten bzw. rot schraffierten Felder visualisiert. Die Lücken sind identifiziert und klein, wobei die Umsetzungstiefe in den verschiedenen Bereichen unterschiedlich ist. Für Open Access beispielsweise wurden nur wenige Mittel nachgefragt. Im Bereich der eLearning-Infrastrukturen wurde erst mit dem Antrag Swiss MOOC Service unter der Leitung der EPFL im Juni 2017 ein Vorhaben bewilligt.

12 SUK P-2: White Paper for a Swiss Information Provisioning and Processing Infrastructure 2020. 2014, hier Kapitel 5.3. Die durchgestrichenen Felder in Abbildung 3 bezeichnen Leerstellen in der Matrix (vgl. Grundlagendokumente, wie Anm. 2). 
Da zahlreichen Projekten in diesem Band ein eigener Beitrag gewidmet ist, beschränkt sich dieser Artikel im Folgenden auf einen selektiven, eher strategischen Bericht aus Sicht der Programmleitung.

\section{„Publikationen“: Bibliotheken im Spannungsfeld zwischen Lizenzierung und Open Access}

Im Programm „Wissenschaftliche Information“ spielt das Konsortium der Schweizer Hochschulbibliotheken eine wenig bekannte Schlüsselrolle: Die prospektiven Beiträge der Hochschulen an das Konsortium können als Eigenmittel zugezogen werden. ${ }^{13}$ Unmittelbar führte dies dazu, dass SUK P-2 das Projekt Nationallizenzen zu 100\% aus Fördermitteln finanzieren konnte. Der Erwerb von Nationallizenzen für digitale Verlagsarchive entsprach einem langjährigen Desiderat des Konsortiums, doch fiel die Umsetzung nun in einen Zeitraum, in dem die Open Access-Bewegung Schub aufnahm. In der Vernehmlassung zu einer Vorversion des White Paper im März 2014 war das Vorhaben sowohl inhaltlich - das Projekt wurde als rückwärtsgewandt eingestuft - als auch aufgrund der absehbar hohen Kosten starker Kritik ausgesetzt, der die Programmleitung bei der Umsetzung später mit einer engen Begleitung Rechnung trug. Die globale Anrechnung der Konsortialbeiträge als Eigenmittel gaben dem Programm im Folgenden aber auch den Freiraum, weitere Projekte, die in der Evaluation als strategisch besonders relevant oder innovativ beurteilt wurden, zu mehr als 50\% zu fördern. Dies gilt im Rahmen der verfügbaren Mittel auch für P-5.

\section{Kollektive Lizenzierung}

Mit den kompletten Zeitschriftenarchiven von De Gruyter, Cambridge sowie University Press und Springer erwarb das Konsortium bis Ende 2016 vier digitale Archive, die den Kriterien entsprachen, die ein externes Beurteilungsgremium für die Nationallizenzen definiert hatte. Zentral war die Umsetzung von Rolling Archives. Diese gewährleisten den nahtlosen Anschluss an die von einzelnen Bibliotheken bereits früher erworbenen Archivbestände und stellen sicher, dass die lizenzierten neuen Inhalte (Current Content) nach einem Embargo in den Archivbestand einfliessen. Zudem wurde eine Green Open Access-Klausel für die publi-

13 Im Antrag für SUK P-2 prospektiv CHF 83 Mio. für die Jahre 2013 bis 2016, im Antrag für P-5 CHF 122,1 Mio. für 2016 bis 2020. 
zierte Version der Archivinhalte verhandelt. Nachhaltige Erfolge des Projekts aus Programmsicht sind auch die breitere Einbindung der Bibliotheken in die Produktediskussion und der Erfahrungsgewinn in den Bereichen Verhandlungs-Setup und Open Access. Die Umsetzung der Metadatenintegration und der Authentifizierungslösung in Zusammenarbeit mit swissbib und SWITCH/Swiss edu-ID stellte zudem interessante Synergien zu zwei weiteren geförderten Projekten her. ${ }^{14}$

\section{Eine Open Access-Strategie für die Schweiz}

Nur geringes Echo fanden die Umsetzungsmassnahmen von SUK P-2 für Open Access. Das Programm unterstützte den Umzug des Journal of Eye Movement Research (JEMR) auf die OJS-Plattform BOP der Universität Bern und den Aufbau der OJS-Plattform HOPE an der Universität Zürich mit dem Journal für Psychoanalyse als Pilotprojekt. Als der Schweizerische Nationalfonds (SNF) im Herbst 2015 die gemeinsame Durchführung einer Finanzflussanalyse zum wissenschaftlichen Publikationswesen in der Schweiz vorschlug, stimmte der Lenkungsausschuss zu. Gleichzeitig rückte Open Access im Zuge der europäischen Initiativen in Richtung Open Science auf der politischen Agenda nach oben. Mit Brief vom 04. Dezember 2015 ersuchte der Staatssekretär SBFI swissuniversities, sich unter Einbezug des SNF ,an der Erarbeitung einer gesamtschweizerischen Strategie für Open Access federführend zu beteiligen“. Er formulierte folgenden Handlungsbedarf:

- gezielte Förderung von Open Access als übergeordnetes Ziel

- Schaffung von Kostentransparenz bei den öffentlichen Ausgaben im Bereich wissenschaftliches Publizieren (Finanzflussanalyse)

- Koordination der Aktivitäten der Stakeholder, seitens der Hochschulen namentlich auch unter Einbezug der Hochschulbibliotheken ${ }^{15}$

Anfang 2016 stiess der Vorstand von swissuniversities den Prozess zur Erarbeitung einer nationalen Open Access-Strategie an. Der Text wurde vom Plenum von swissuniversities am 31. Januar 2017 verabschiedet und vom Hochschulrat der SHK am 23. Februar zur Kenntnis genommen, unter Vorbehalt eines Aktionsplans, der ergänzend zur Strategie entsteht. Bereits im November 2016 hatte die Studie Financial Flows in Swiss Publishing, basierend auf ökonomischen Modellierungen und

14 Vgl. http://www.consortium.ch/national-lizenzen/.

$15 \mathrm{Vgl}$. https://www.swissuniversities.ch/de/themen/hochschulpolitik/open-access/. Die Seite dokumentiert den Entstehungsprozess und die Resultate der Open Access-Strategie. Im Einzelnen handelt es sich um den hier verlinkten Brief des SBFI (Staatssekretär Mauro Dell'Ambrogio) an das Präsidium von swissuniversities (Prof. Dr. Martine Rahier) vom 04.12.2015. 
einer Erhebung bei den Hochschulen, einen ersten Beitrag zur geforderten Kostentransparenz geleistet, indem sie für die Schweiz erstmals Zahlen und Szenarien zum Verhältnis der traditionellen Publikationskanäle und Open Access lieferte. Ergänzend erstellte die Max Planck Digital Library eine bibliometrische Analyse. ${ }^{16}$

Die Umsetzungsstrategie 2017 bis 2020 trägt diesen Entwicklungen Rechnung. Auf ihrer Grundlage unterstützt P-5 sowohl die Ausarbeitung des Aktionsplans zur Open Access-Strategie durch swissuniversities als auch eine Stelle zum Aufbau von Open Access-Kompetenz beim Konsortium mit 50\%. Es besteht Hoffnung, dass dadurch eine bessere Basis für kollektive Projekte entsteht. Die Hauptstossrichtung „Publikationen“ schlägt eine Reihe weiterer Massnahmen zur Förderung von Open Access-Content und -Infrastrukturen vor - last but not least den weiteren Ausbau des Konsortiums für den kollektiven Erwerb. Unterstützen die Hochschulen ein stärkeres Konsortium, u.a. mit mehr Verhandlungsmacht gegenüber den grossen Wissenschaftsverlagen, kann die Transformation des Marktes in Richtung Open Access auch von der Schweiz mit angeschoben werden.

\section{„Publikationen“: Digitalisierung, quo vadis?}

Auf den ersten Eingabetermin von SUK P-2 im Frühling 2014 gingen sieben Anträge zur Umsetzungsmassnahme EP-10 ein, deren Förderzweck lautete: „Die Digitalisierung von Inhalten von nationaler Relevanz auf einer bestehenden, teilnehmeroffenen Digitalisierungsplattform (retro-seals, e-manuscripta.ch, e-rara.ch, Scriptorium, rero.doc u.a.m.). Die Betreiber der Digitalisierungsplattformen bieten ihren Service zu Vollkosten und mit einer Servicevereinbarung (SLA) an. “ ${ }^{\text {"17 }}$

Die Schwierigkeit von Experten und Gutachtern, die ,nationale Relevanz'von Inhalten zu beurteilen, die von den Antragstellern - hauptsächlich Bibliotheken - in eigener Regie zur Retrodigitalisierung vorgeschlagen wurden, führte da$\mathrm{zu}$, dass die Anträge zurückgestellt und nach der Erarbeitung einer dedizierten Strategie zum Thema Digitalisierung schliesslich abgewiesen wurden. ${ }^{18}$ Die angepasste Strategie konkretisierte im März 2015 den Wunsch nach einer breiteren Partizipation von Gedächtnisinstitutionen und Forschung an den Plattformen, die

16 Alexander Machado, Laura Hoppmann u.a.: Bibliometric study of the Swiss Publication System. 18. November 2016. Zenodo: http://doi.org/10.5281/zenodo.167381.

17 SUK P-2: White Paper for a Swiss Information Provisioning and Processing Infrastructure 2020. 2014, S. 30.

18 SUK P-2: Hauptstossrichtung Publikationen, Umsetzungsmassnahme EP-10, Digitalisierung: angepasste Strategie und Umsetzungsmassnahmen vom 31.03.2015 (vgl. Grundlagendokumente, wie Anm. 2). 
z.B. im Rahmen von e-lib.ch aufgebaut worden waren. Die Verbesserung der Interoperabilität (,from silos to strata“), die Implementierung von Standards (technische Standards und Metadaten) und die Entwicklung von Tools für die Forschung traten gegenüber der Retrodigitalisierung von Inhalten in den Vordergrund.

Die Erarbeitung des Strategiedokumentes erfolgte zeitgleich mit einem Call für Editionsprojekte des SNF sowie einem Pilotprojekt der Schweizerischen Akademie der Geistes- und Sozialwissenschaften (SAGW) und der Universität Basel zur Einrichtung eines Daten- und Dienstleistungszentrums für die Geisteswissenschaften (Data and Service Center for the Humanities, DaSCH). ${ }^{19}$ Der Austausch mit diesen Vorhaben machte Anforderungen an die Digitalisierung von Seiten der Digital Humanities deutlich. Mit dem Projekt NIE-INE (Nationale Infrastruktur für Editionen), das Mitte 2016 bewilligt wurde, fördert das Programm deshalb ein Kooperationsvorhaben der Universitäten Basel, Zürich, Bern und Genf sowie der SAGW zum Aufbau einer technischen Architektur für die Bearbeitung digitaler kritischer Editionen. ${ }^{20}$ Im Projekt histHub erarbeiten vier Unternehmen der SAGW einen Hub für historische Entitäten als Linked Open Data. Eine Schweizer Spezialität wird die Mehrsprachigkeit der Vokabulare sein.

Insgesamt verlagerte sich die Anschubfinanzierung im Umsetzungsbereich Digitalisierung vom Zielgebiet Bibliotheken in Richtung Digital Humanities. Auch die Ausbauprojekte der Plattformen e-manuscripta.ch (für digitalisierte handschriftliche Quellen aus Schweizer Bibliotheken) und e-rara.ch (für digitalisierte Drucke aus Schweizer Bibliotheken) zielen in Richtung Öffnung der Quellen für Forschende. Im Februar 2017 hat die HTW Chur eine Analyse der Informationsarchitektur, Schnittstellen und Organisation der Online-Plattformen vorgelegt. ${ }^{21}$ $\mathrm{Ob}$ auf dieser Grundlage neue Projekte entstehen, ist offen. Die Virtuelle Handschriftenbibliothek Schweiz (e-codices) wird in der Phase P-5 beim Aufbau einer eigenständigen Trägerschaft unterstützt.

Die Umsetzungsmassnahmen der angepassten Strategie Digitalisierung wurden in der Umsetzungsstrategie 2017 bis 2020 für P-5 unverändert übernommen. Mehrere Massnahmen stiessen bisher auf kein Interesse, darunter die Idee, einen kollektiven Fonds zur Finanzierung von Retrodigitalisierung zu gründen. Die Bildung eines Kompetenzzentrums Digitalisierung scheiterte vorerst im zweiten Anlauf.

19 Zum DaSCH vgl. http://www.sagw.ch/sagw/die-akademie/unternehmen/DaSCH.html.

20 Die Projektleitung hat das Forum Edition und Erschliessung (FEE) der Universität Basel (http://www.fee.unibas.ch/nie_ine.html).

21 Projekt AISOOP, für den Bericht vgl. https://www.swissuniversities.ch/fileadmin/swissuni versities/Dokumente/Organisation/SUK-P/SUK_P-2/BerichtAISOOP_final.pdf. 


\section{"eScience": Bibliotheken als Anbieter und Kunden im Forschungsdatenmanagement}

Im Kontext von Open Science - und in konsequenter Erweiterung der Open Access-Policy - verlangt der SNF seit Oktober 2017 als integralen Bestandteil eines Forschungsgesuchs einen Datenmanagementplan (DMP). Im Sinne der Transparenz und Reproduzierbarkeit von Forschung sollen Forschungsdaten sorgfältig aufbereitet, archiviert und mit dem Ziel einer grösseren Wirkung auch möglichst zugänglich sein. Mindestens die Daten, die einer Veröffentlichung zugrundeliegen, sollen offen zur Verfügung gestellt werden. ${ }^{22}$

Die Forderung nach Open Data setzt somit voraus, dass das Data Lifecycle Management im Hintergrund geregelt ist. Die Supportanfragen im Nachgang der Kommunikation des SNF zeigen, dass die Forschenden, die Schweizer Hochschulen und ihre Dienste diesbezüglich unterschiedlich gut aufgestellt sind. In weiten Bereichen ist Data Lifecycle Management noch immer Neuland. Dies gilt nicht nur für die Infrastrukturen, sondern auch für Sensibilisierung, Knowhow und Beratung.

\section{Data Life-Cycle Management (DLCM)}

In diese Lücke stösst das Projekt DLCM. Es will Konzepte, Materialien und exemplarische Lösungen zur Unterstützung des Lebenszyklus von Forschungsdaten erarbeiten, die allen Hochschulen zur Verfügung stehen. Das Projekt, in das bis Mitte 2018 mehr als CHF 6 Mio. Programmmittel fliessen, ist breit abgestützt. Die Partner - die Eidgenössischen Technischen Hochschulen, die Universitäten Genf, Lausanne, Basel und Zürich, die Fachhochschule Genf und SWITCH - entwickeln die Inhalte in einem Bogen über die West- und Deutschschweiz, die Geistes- und Naturwissenschaften sowie IT-Services, Scientific IT und Bibliotheken. ${ }^{23}$ Der Aktionsschwerpunkt der Bibliotheken (EPFL, ETHZ, Universitäten Genf und Zürich) ist die Sichtung internationaler Quellen und Best Practice-Beispiele zur Nachnutzung in der Schweiz, die Erstellung von Vorlagen (Templates) und Richtlinien für Data Management-Policies und -Pläne, aber auch die Aufbereitung der Materialien im gemeinsamen Portal des Projekts.

22 SNF. Data Management Plan (DMP) - Leitlinien für Forschende (http://www.snf.ch/de/der Snf/forschungspolitische_positionen/open_research_data/Seiten/data-management-plan-dmp-1 eitlinien-fuer-forschende.aspx).

23 Projektseite: http://www.dlcm.ch/. 
Trotz der guten Ausgangslage steht DLCM exemplarisch für das Verbesserungspotenzial bei der Steuerung und Unterstützung von Kooperationsprojekten: 2016 erarbeitete DLCM eine Vorlage für die Erarbeitung der Forschungsdaten-Policy einer Hochschule. Ein Versuch, die Vorlage den Hochschulen via swissuniversities empfehlen zu lassen, schlug 2016 vorerst fehl. DLCM und SNF erarbeiteten unabhängig voneinander Vorlagen für einen Datenmanagementplan. Als der SNF im März 2017 die neuen Anforderungen betreffend Forschungsdatenmanagement kommunizierte und zahlreiche Supportanfragen auslöste, war DLCM darauf nicht vorbereitet. In der Folge war zu beobachten, wie die DLCMPartner nun zunächst den lokalen Support aufrüsteten. Der Aufbau des nationalen Angebots rückte trotz der Dringlichkeit für alle vorerst in den Hintergrund.

Auf Initiative des SNF und im Beisein der Programmleitung P-5 finden seit dem Sommer 2017 periodisch Treffen mit DLCM statt. Unmittelbar geht es darum, wie DLCM die Forschenden bei der Erfüllung der SNF-Auflagen unterstützen kann. Im grösseren Zusammenhang ist P-5 an einer besseren Verbindung von Policy- und Infrastrukturprojekten und an der breiteren Verankerung wichtiger Kooperationsprojekte interessiert.

\section{DLCM-Training und Train2Dacar}

Im Projekt DLCM arbeiten Bibliotheken als Anbieter an neuen Dienstleistungen für das Forschungsdatenmanagement. Da Forschungsdatenmanagement auch für Bibliotheken Neuland ist, sind sie selbst wiederum Kunden für Ausbildungsund Trainingsangebote. Die Erarbeitung und Bereitstellung von Trainingsmodulen ist auch als Teil von DLCM vorgesehen. Bereits seit Frühling 2017 steht zudem die E-Learning-Plattform Forschungsdatenmanagement des Projekts Train2Dacar im Internet. ${ }^{24}$ Die Lernmodule, die an den Fachhochschulen Genf und Chur entstanden, ermöglichen Forschenden und Mitarbeitenden von Bibliotheken und ITServices eine Weiterbildung in Data Management-Support.

\section{„Basis“: SLSP als Bibliothekshub im künftigen Servicenetzwerk}

Am 30. Mai 2017 gründeten 15 Institutionen mit Bibliotheken aus dem Hochschulbereich die Aktiengesellschaft SLSP Swiss Library Service Platform AG. Unmittel-

24 Train2Dacar. Research Data Management. E-Learning Platform. http://www.researchdata management.ch/ (alle Module sind auf deutsch und französisch verfügbar). 
bar danach wurde die Beschaffung eines gemeinsamen Bibliothekssystems eingeleitet. ${ }^{25}$ Der Austritt des Kantons Waadt aus dem Bibliotheksverbund RERO und der Umstand, dass die integrierten Bibliothekssysteme der grossen Hochschulbibliotheken das Ende ihres Lebenszyklus erreichten, hatte 2014 unter dem Lead der ETH-Bibliothek zur breit abgestützten Initiative geführt, die Schweizer Bibliotheksverbünde technisch und organisatorisch auf neue Grundlagen zu stellen. Mit SLSP entsteht rund um ein integriertes Bibliotheksverwaltungs- und Discovery-System der neuen Generation eine Serviceplattform, die Schweizer Bibliotheken ein breites Spektrum von Dienstleistungen anbietet.

SLSP wurde der Hauptstossrichtung „Basis“ des Programms zugeordnet, in der die technischen und organisatorischen Grundlagen für die Zusammenarbeit von Bibliotheken, IT-Services und Scientific IT aufgebaut werden. Für „Wissenschaftliche Information“ ist SLSP ein Projekt, das herausfordert:

- Obwohl SLSP erst nach dem Strategieprozess für das Programm am Horizont auftauchte, ist es heute ein Schlüsselprojekt im Portfolio. Es belegt, dass der kontinuierliche und dynamische Aufbau eines nationalen Servicenetzwerks auch ,unterwegs‘ finanziellen Spielraum bedingt. Von August 2015 bis Februar 2017 erhält SLSP vorerst Fördermittel in Höhe von CHF 1,8 Mio.

- Förderentscheide müssen jedoch strategie- und regelkonform gefällt werden. Gemäss Strategie unterstützt P-5 die Investitionskosten für die Öffnung von Systemen für mehr Teilnehmer bzw. die Zutrittskosten neuer Teilnehmer zu bestehenden Systemen. Projektgebundene Beiträge sind jedoch nicht für die sowieso fällige Erneuerung von Systemen gedacht. Projektanträge werden zudem in einem Evaluationsverfahren geprüft. Diese Grundlagen gelten auch für SLSP.

- SLSP stellt die Gremien von swissuniversities - die Gesamtheit der Schweizer Hochschulen - vor einen Interessenkonflikt. Die Hochschulen können daran interessiert sein, die Erneuerungskosten für Bibliothekssysteme aus der Kasse von P-5 zu decken. Gleichzeitig sind die Hochschulen die Auftraggeber des Programms „Wissenschaftliche Information“ und die Erreichung der Programmziele bedingt, dass sie die Verfolgung der Strategie mit glaubwürdigen, nachvollziehbaren Projektentscheiden unterstützen. Ob die Programmstrategie diesem Interessenkonflikt standhält, ist für das Gesamtvorhaben des Programms richtungsweisend.

- Die Gründung der SLSP AG hat die Hochschulen aufgeschreckt und sie auf zahlreiche Organisationsfragen aufmerksam gemacht, die sich auch bei weiteren Kooperationsprojekten sowie der geplanten Koordinationsstelle für das

25 Vgl. „Die SLSP AG ist gegründet!“ vom 30.05.2017 auf https://blogs.ethz.ch/slsp/. 
Servicenetzwerk stellen. Wieweit die Hochschulen dazu bereit sind, sich auf diese Diskussion einzulassen und sich den Herausforderungen vermehrter Kooperation zu stellen, ist offen.

Bei erfolgreicher Umsetzung von SLSP erhält die Schweiz einen nationalen Hub für Bibliotheksdienstleistungen, der mit SWITCH im Bereich IT-Services vergleichbar ist. SLSP soll künftig das Konsortium der Schweizer Hochschulbibliotheken und den Metadatenhub der Schweizer Bibliotheken swissbib integrieren. Weiteres Integrationspotenzial besteht bei den Digitalisierungsplattformen oder bei Diensten, die im Kontext der Open Science-Strategie von SNF und swissuniversities gefördert werden. Synergien mit dem CCdigitallaw, das rechtliche Unterstützung in den Bereichen Urheber- und Datenrecht aufbaut, sind ebenfalls denkbar. Von einer Zusammenarbeit mit der Kooperativen Speicherbibliothek Schweiz ist auszugehen.

\section{„Basis“: Aufbau einer Drehscheibe für teilnehmeroffene Dienste und Infrastrukturen}

In der Phase SUK P-2 ist es dem Programm „Wissenschaftliche Information“ gelungen, die Dienstleister der Hochschulen (Bibliotheken, IT-Services und Scientific IT) zu vernetzen und in starken Kollaborationen zusammen zu bringen. Ende 2016 leiteten die Hälfte aller universitären Hochschulen und zwei von sieben Fachhochschulen eines oder mehrere Projekte. Alle kantonalen Universitäten, beide ETHs sowie sechs von sieben Fachhochschulen waren an Projekten beteiligt. Seit Mitte 2017 sind zudem alle Mitglieder des Lenkungsausschusses des Programms von den drei Kammern von swissuniversities, dem Netzwerk der ITServices der Hochschulen sowie der Konferenz der Universitätsbibliotheken der Schweiz (KUB) delegiert. Damit ist die Voraussetzung für eine engere strategische Anbindung gegeben. Ob die Dynamik aufrechterhalten werden kann, wird sich in den Jahren 2017/2018 zeigen, in denen gleich mehrere Hürden zu nehmen sind.

\section{Unterstützung einer nationalen Governance}

Die nationale Strategie für SUK P-2 bezeichnet die Programmorganisation als Ausgangspunkt für den Aufbau einer dauerhaften Koordinationsstelle und einer Serviceplattform für die aufgebauten Dienste. Diese Koordinationsstelle soll den Servicekatalog führen, die Einhaltung von Vereinbarungen im Servicenetzwerk überprüfen, Richtlinien und Schnittstellen definieren und den Einsatz von Finanz- 
mitteln koordinieren. ${ }^{26}$ Die Arbeit am Aufbau dieser Stelle begann in der zweiten Hälfte 2016 und im Mai 2017 wurde ein Grobkonzept in Konsultation gegeben. ${ }^{27}$ Die verhaltenen Reaktionen der Stakeholder zeigten, dass die Vorlage für eine produktive Diskussion nicht konkret genug war. Die erst vage Beschreibung der Aufgaben dieser Stelle führte zu sehr unterschiedlichen Äusserungen sowohl in Bezug auf deren Notwendigkeit als auch in Bezug auf deren Verortung. Einig war man sich grosso modo, dass ein vermehrt koordiniertes Vorgehen bei gemeinsamer strategischer Ausrichtung vermutlich sinnvoll wäre und dass die Governance einer solchen Stelle bei swissuniversities angesiedelt sein müsste. Im Herbst 2017 soll nun der Auftrag des Programms bei swissuniversities noch einmal rückversichert werden. Zudem soll swissuniversities sich vor der Erarbeitung eines Detailkonzeptes zur grundsätzlichen Ansiedlung der Koordinationsstelle äussern. Gemäss Konsultation kommen für die Wahrnehmung der operativen Aufgaben in erster Linie SWITCH oder swissuniversities selbst in Frage. Der Aufbau einer völlig neuen Organisation steht im Hintergrund. Der Entscheid der Hochschulen für ein Szenario soll es der Programmorganisation ermöglichen, 2018 unter bekannten Rahmenbedingungen ein Detailkonzept zu erarbeiten, das ,Hand und Fuss“ hat. Ziel ist, dass swissuniversities das Vorhaben Ende 2018 in die Planung für die BFI-Botschaft 2021-2024 einbringt.

\section{Nachweis erfolgreicher Dienste}

Für die Legitimation einer nationalen Governance mit Koordinationsstelle hilft der Verweis auf eine Anzahl stabiler Dienste, die heute bereits zum Servicenetzwerk gehören. Aus Sicht der Programmleitung zählen die folgenden Angebote zum ,Startkapital': ${ }^{28}$

- „Publikationen“: Nationallizenzen, HOPE, e-codices, e-rara.ch, e-manuscripta.ch, swissbib

- „eScience“: EnhanceR, PSI Data Analysis Service, Train2Dacar

- „Basis“: SWITCHengines/SCAL-UP, SWITCH edu-ID, National e-Infrastructure Link, CCdigitallaw

- „Dienste“: Kooperative Speicherbibliothek Schweiz, geodata4edu.ch

26 SUK P-2: Nationale Strategie. 2014, hier Kapitel 1.5 und 4. Die Grundlagen des Betriebsmodells wurden im White Paper, Kapitel 4.7, erarbeitet (vgl. Grundlagendokumente, wie Anm. 2).

27 P-5: Projekt „nationale Organisation“ (Arbeitstitel). Grobkonzept für Aufgabenbereich, Struktur und Aufbau. 2. Mai 2017 (vgl. Grundlagendokumente, wie Anm. 2).

28 Eine Übersicht über die geförderten Dienste befindet sich auf der Programmwebseite (vgl. Anm. 2). 
Die Anforderung, bereits erfolgreiche, selbsttragende Dienste nachzuweisen, ist hoch. Die Dienste, die vom Programm angeschoben wurden, sind erst im Begriff, sich auf eigene Beine zu stellen, die Gründung einer nachhaltigen Organisationsform ist anspruchsvoll und wird von den Hochschulen bedingt unterstützt. Die Koordinationsstelle, die als Anknüpfungspunkt dienen könnte, ist noch nicht vorhanden. Zudem besitzt das Programm keine eigentliche Legitimation, einmal geförderte Dienste - oder schon länger etablierte Dienste, die Mittel aus dem Programm erhalten haben - in ein Servicenetzwerk einzubinden. Dies ist nur möglich, wenn alle beteiligten Parteien einen Mehrwert erkennen. Das Programm betritt hier Neuland. Mit dem Slogan „mandated by swissuniversities“ wird ein erster Versuch gemacht, die Dienste als unter einem gemeinsamen Label stehend kenntlich zu machen. Mit der Lancierung eines professionellen Servicemanagements gemeinsam mit einem Team der Hochschule Rapperswil hat die Programmorganisation 2017 damit begonnen, erste gemeinsame Bezugspunkte für das Netzwerk herauszuarbeiten. Im künftigen Servicenetzwerk soll die Koordinationsstelle als ,Enabler' sowohl für die Erbringung als auch für den Bezug von Diensten unter den Hochschulen auftreten.

\section{Fokussierung auf gemeinsame strategische Vorhaben}

Im föderalistischen Hochschulwesen der Schweiz hängt der Erfolg des Programms „Wissenschaftliche Information“ wesentlich davon ab, ob die Massnahmen, die in die Umsetzungsstrategie aufgenommen wurden, sich mit eigenen Vorhaben bzw. Interessen der Hochschulen decken. Nur dann finden sich Anbieter und Abnehmer für Dienste und Infrastrukturen und nur dann sind die Hochschulen dazu bereit, gemeinsam in das Vorhaben zu investieren.

Dies wird ab 2021 noch mehr gelten. Findet die Einrichtung einer Koordinationsstelle für den Betrieb eines Servicenetzwerks Unterstützung, müssten bis Ende 2018 auch die strategischen Bereiche eingezirkelt werden, in welche die Hochschulen mithilfe dieser Organisation in der nächsten Finanzierungsperiode investieren wollen. Bleibende Themen aus heutiger Sicht sind Lösungen für das Forschungsdatenmanagement (u.a. Repositories) und die Unterstützung von Open Access bzw. neuer Publikationsmodelle.

Über eine nationale Koordinationsstelle könnten in Zukunft auch die Anforderungen der Forschungsförderorganisationen und die Entwicklung der Infrastrukturen an den Hochschulen besser aufeinander abgestimmt werden. Soll das Programm „Wissenschaftliche Information“ die beabsichtigte Portfoliobereinigung bewirken, erfordert dies in Zukunft eine engere Zusammenarbeit auf der Ebene der Schweizer Hochschulplanung. Dabei ist das Verhältnis der Koordina- 
tionsstelle zu Dienstleistungsanbietern wie SWITCH, SLSP, der Schweizerischen Akademie der Geistes- und Sozialwissenschaften (SAGW) oder dem künftigen Swiss Data Science Center (SDSC) zu klären.

\section{Die Schweizer Bibliotheken als Dienstleister für alle}

Offen ist, wie sich durch die Bibliotheksdienste, die das Programm anschiebt insbesondere durch SLSP - die Dienstleistungen des schweizerischen Bibliothekswesens insgesamt entwickeln. „Wissenschaftliche Information“ fördert gemeinsame Lösungen im Hochschulbereich und wirkt deshalb zunächst im wissenschaftlichen Bibliothekswesen. In der Schweiz sind die Übergänge zwischen den Wissenschaftlichen Bibliotheken und den Bibliotheken der Kantone, Städte, Gemeinden und Schulen jedoch fliessend: Das System zeichnet sich dadurch aus, dass alle Bibliothekstypen weitgehend öffentlich sind und über die Grenzen des föderalistisch geregelten Kultur- und Bildungsbereichs der Schweiz hinweg komplementär eine Gesamtdienstleistung erbringen.

Citizen Science können die Schweizer Bibliotheken also schon lange. Die neuen Dienste der Wissenschaftlichen Bibliotheken sollten die Sichtbarkeit und die Zugänglichkeit von Beständen in Zukunft weiter verbessern. Während Open Access jedem und jeder den unmittelbaren Zugriff auf wissenschaftliche Publikationen ermöglicht, kann die Swiss edu-ID à la Nationallizenzen den mobileren Zugang zu lizenzierten Inhalten unterstützen. Es besteht auch Hoffnung, dass die Nationallizenzen ein Stück des Wegs zu flächendeckenderen Lizenzmodellen geebnet haben.

Das Beispiel SLSP zeigt, dass auch unterschiedliche Anforderungen sowie rechtliche und finanzielle Zuständigkeiten zu verhandeln sind. Auch wenn am Ende nicht jede Bibliothek Teil von SLSP ist: Die Einführung von Normen und standardisierten Schnittstellen, zusammen mit den genannten Errungenschaften, wird die Zusammenarbeit verschiedener Systeme und Effizienzgewinne im Gesamtsystem zweifellos begünstigen. 